\title{
THE USE OF INSTRUMENTS IN COMMISSUROTOMY FOR MITRAL STENOSIS

\author{
BY
}

\author{
J. NAUTA AND H. HARTMAN \\ From the Departments of Thoracic Surgery and Cardiology of the Leyden University Hospital, \\ Leyden, the Netherlands
}

(RECEIVED FOR PUBLICATION MARCH 24, 1961)

This study was made in order to establish the effect of the use of instruments during commissurotomy for mitral stenosis. Initially, the adherent commissures between the mitral valves were severed exclusively with the finger, because the digital method had the advantage that possible adhesions between the papillary muscles could be detached with the finger. Soon it was found, however, that a good result could not be obtained in all cases; for example, the adhesions between the valves were sometimes too firm to permit of digital severance. In other cases the commissures were readily severed but the finger caused incompetence (Ellis, Harken, and Black, 1959 ; Bailey and others, 1959).

In the case of exclusively digital commissurotomy, therefore, it is possible to distinguish two groups of patients in whom the operation cannot be considered sufficiently successful, viz., (1) patients in whom the orifice cannot be sufficiently opened during the operation, and (2) patients who develop an incompetence which did not previously exist or existed in a lesser degree than after the operation.

It is understandable that various instruments were introduced to overcome the above shortcomings of digital commissurotomy, and it would be desirable to know which instruments are best suited to this operation. It is as yet impossible to establish this with certainty because comparative series are still lacking. During the operation every surgeon has the choice of a variety of instruments; in some cases he will use none of these ; in others he will use instrument $A$ or instrument $B$, dependent on his own experience and preference and on the intracardiac situation which he encounters. Only few have so far managed, like Gerbode (1960), to treat a continuous series of patients by the same technique and subsequently to analyse the results.

In our clinic the use of instruments has gradually increased. It is our intention to establish in this paper the result of this gradual change in technique in terms of: (1) the width of the mitral orifice obtained; (2) the occurrence of incompetence as a result of the operation. Later we intend to establish the importance of these two factors for the post-operative years of our patients.

\section{METHOD}

The investigation concerned 310 patients who were operated on during the period 1954-59. During every operation both the surgeon and his assistant palpated the mitral orifice, so that optimal objectivity was ensured in estimating the width of the orifice and the degree of incompetence. This estimate was made both before and after the commissurotomy, and recorded in the operation report. As the orifice was palpated with the right index finger, its width was estimated by finding, with the left index finger, an opening of the same diameter in a perforated metal plate. The degree of incompetence was palpated with the finger and classified as follows:

Grade 0-1: incompetence only just palpable

Grade 1: slight but unmistakable incompetence

Grade 2: moderately marked incompetence

Grade 3: marked incompetence

Grade 4: catastrophic incompetence

Sometimes neither the surgeon nor his assistant palpated incompetence after the intervention. In some of these cases, however, subsequent examination at the out-patient clinic revealed a systolic souffle at the apex of the heart, from which we concluded that slight mitral incompetence none the less existed. The phonocardiogram was obtained with the aid of fouror five-channel Hellige Multikardiotest type $\mathbf{9 9 0 0 .}$

The patient's state before and after the operation was estimated as suggested by the New York Heart Association (1953).

\section{RESUlTS}

During the period 1954-59 some 50 patients annually underwent an operation for mitral stenosis. In 1954 digital commissurotomy was performed in $80 \%$ of cases; an instrument was 


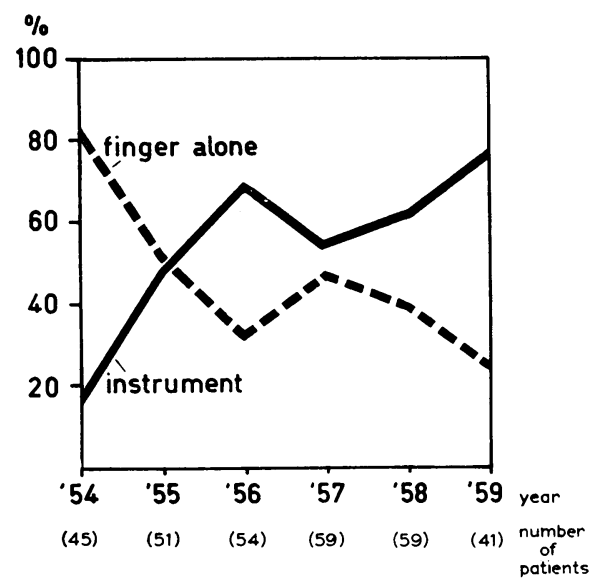

FIG. 1.-Graph illustrating the increasing use of instruments in commissurotomy between 1954 and 1959.

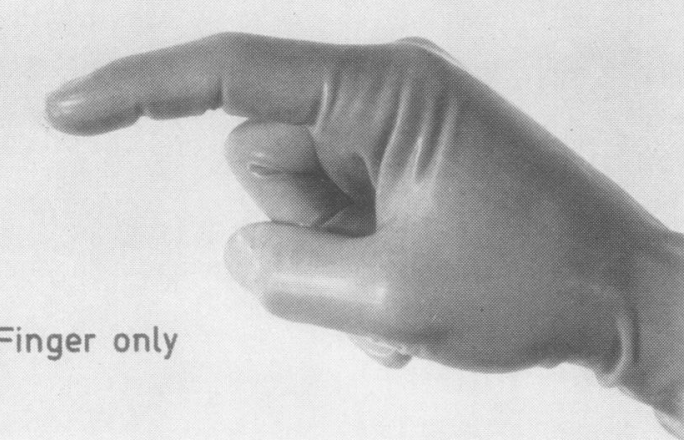

\section{Brock's knife} ant. lat commiss.

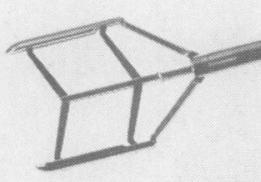

Tubbs's dilator

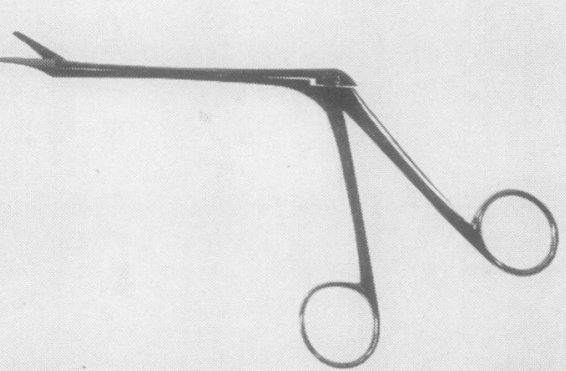

used in $20 \%$. In the course of the subsequent years the use of instruments continuously increased, and in 1959 only $20 \%$ of commissurotomies were digital, whereas $80 \%$ were performed with the aid of an instrument (Fig. 1).

The instruments used in the course of these years were the following (Fig. 2).

A Brock knife, which was introduced, together with the right index finger, via the left auricle, and used to sever the antero-lateral commissure.

Modified Struycken scissors, introduced into the left atrium through a separate opening and, guided by the finger, used to sever the postero-lateral commissure (Romanos and Derom, 1955).

A Tubbs dilator, which was always introduced via the apex of the heart. As the dilator was opened, its "arms" were lying in the commissures (Logan and Turner, 1959; Dubost, 1954).

Fig. 3* shows that the number of digital commissurotomies steadily decreased. The Brock knife was used with about constant frequency in

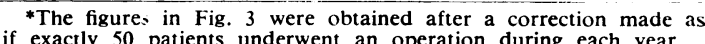
if exactly 50 patients underwent an operation during each year.

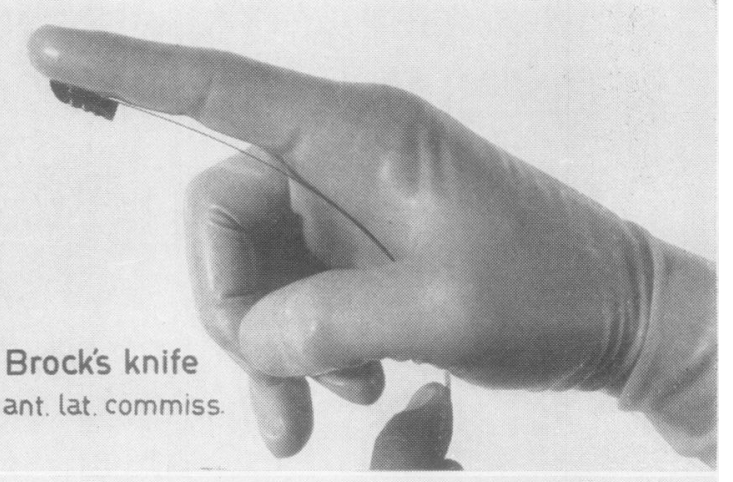

post. med. commiss.

FIG. 2.-Techniques used in commissurotomy during the period 1954-59. 
finger alone Brock knife scissors dilator
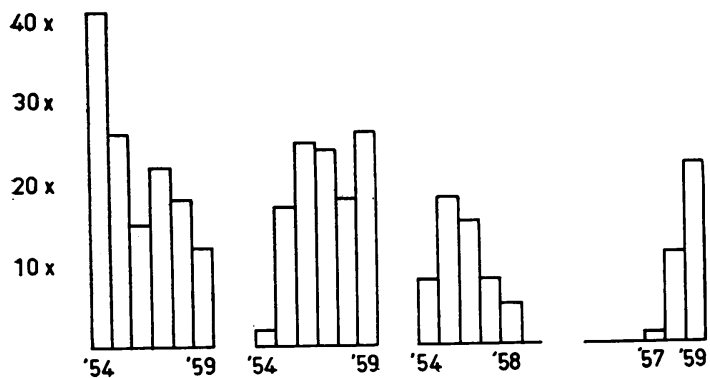

F1G. 3.-Diagram illustrating decrease in number of exclusively digital commissurotomies in 1954-59. The use of a dilator has increased since 1957. The scissors were abandoned in 1958. The Brock knife was used equally often in all years.

the years after 1954. Struycken scissors were seldom used in 1958 and no longer in 1959. The Tubbs dilator has been increasingly used in the past few years.

Result in Terms of Orifice Width.-Considering, in this discussion, the number of patients in whom the mitral orifice was insufficiently opened, we must eliminate those with postoperative incompetence exceeding grade 1 . In the majority of these cases the surgeon was capable of increasing the width of the orifice but refrained from doing so in view of the possibility of a dangerous increase in incompetence. As was to be expected, the use of instruments has reduced the incidence of small orifices after the operation. The fact that the curve in Fig. 4 fails to fall to

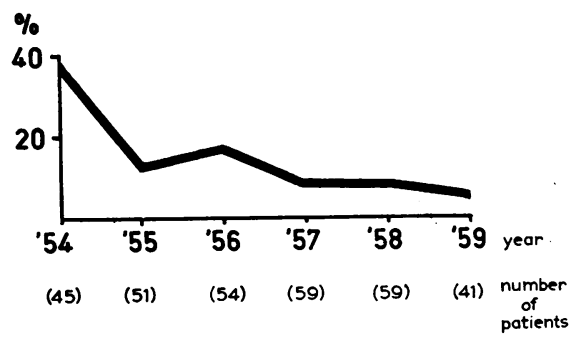

FIG. 4.-The percentage of patients with a small post-operative mitral orifice decreased as a result of the use of instruments in commissurotomy.

$0 \%$ is explained by the circumstance that, in a small percentage of patients, valves were found to be so fibrotic and calcified as to preclude the making of a wide orifice, at least by a blind operative technique.

Result in TeRms of OccurRence of Valvular INCOMPETENCE.-The development of incompetence as a result of the operation may mean either that previously entirely competent valves became incompetent after the operation, or that a previously existing incompetence was exacerbated. The top left curve in Fig. 5 shows that every year some incompetence occurred in 30 to $40 \%$ of patients treated by digital commissurotomy. This in spite of the fact that the indication for digital commissurotomy was accepted less and less often, and finally only in the so-called "easy" cases. The top right curve in Fig. 5 shows a similar phenomenon in the cases in which the commissures were severed with the aid of an instrument. The fact that this line shows a favourable downward trend (from 50 to $30 \%$ ) is not necessarily significant.

In general, the operating surgeon estimated the post-operative incompetence to be more marked than that before the operation in 30 to $40 \%$ of patients. This does not imply that incompetence was always severe and of haemodynamic importance. Further analysis showed that incompetence was inflicted as often on patients with previously competent as on those with previously incompetent valves. Presuming that an incompetence exceeding grade 1 can have unfavourable sequelae for the patient, we ascertained how many patients were discharged with this degree of incompetence (not previously existing). The result of this investigation is shown in the bottom curves of Fig. 5. Incompetence exceeding grade 1 existed after digital commissurotomy in 15 to $20 \%$ of cases. After instrumental commissurotomy this figure was initially larger $(20-30 \%)$, but it decreased to $6 \%$ in 1959.

The question as to how many patients showed incompetence exceeding grade 1 after an operation, regardless of the operative technique, is answered in Fig. 6. The 1954 figure was 12\% ; this rose to $20 \%$ in 1955 as a result of the trend in favour of wider orifices and the use of instruments, and fell to $7 \%$ in 1959 . The progress made in 1955 by attaining a wider orifice was associated with a loss due to the increased frequency of incompetence. Only in 1959 did a favourable situation develop, in which wide orifices were made while marked incompetence was rare.

We believe that this progress has been chiefly due to an ever more frequent use of the Tubbs dilator and abandonment of the Struycken scissors. Certainty in this respect, however, can only be obtained as much larger series of cases become available. We also tried to estimate which of the following three factors was of importance to the patient :

Valvular Mobility.-It is possible that the effect of a successful commissurotomy can be 

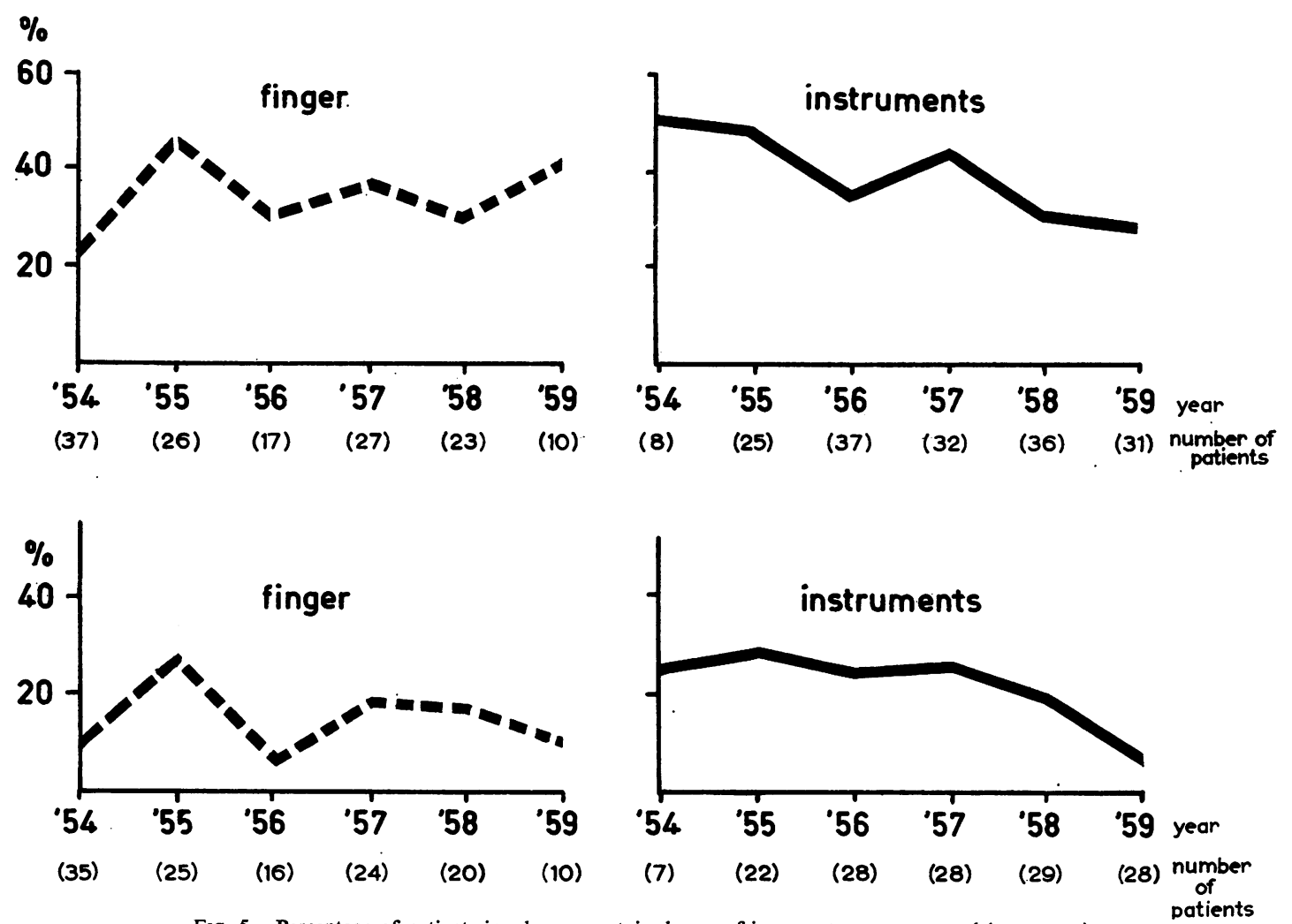

FIG. 5.-Percentage of patients in whom a certain degree of incompetence was caused (top curves). Bottom curves show percentage with incompetence exceeding grade 1 after operation.

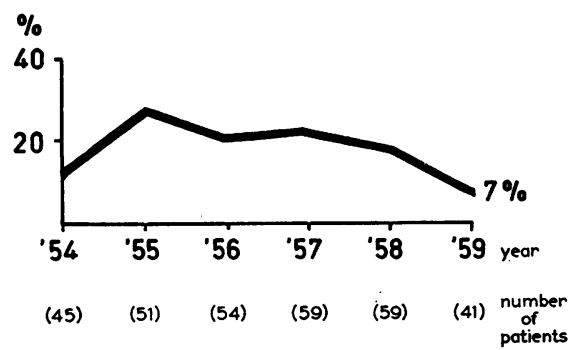

FIG. 6.-A survey of all patients treated by operation during : 1954-59 $\rightarrow$ reveals an important decrease in the percentage of incompetence exceeding grade 1.

destroyed by the presence of severely calcified, immobile valves. After all, these might turn the orifice into an immobile fissure, thus causing persistence of the functional stenosis. Consequently it is worth while establishing whether a correlation exists between the clinical postoperative course and the degree of valvular mobility.

WidTh OF THE Mitral ORIFICE.-An orifice of 2 to 3 sq. $\mathrm{cm}$. after the operation must be considered too small and thus constitutes an unsatisfactory result. Surgeons, however, are confronted with the following problem. Is maximal severance of the commissures an absolute necessity, or can a submaximal orifice be regarded as acceptable ? In other words, Is it of importance to the patient that the last half-centimetre of the commissure is also open ?

Degree of Valvular Incompetence.-In the course of a commissurotomy the surgeon may be confronted with a situation in which the orifice is not yet at its maximal opening while marked incompetence already exists. The surgeon's choice is then between refraining from further widening the orifice and continuing the commissurotomy, accepting the risk of increasing incompetence. To evaluate the significance of the degree of incompetence, an attempt was made at correlating it with the post-operative clinical course.

The influence of these three factors, valvular mobility, maximal or non-maximal widening of the mitral orifice, and the degree of incompetence, on the clinical course is shown in Fig. 7. 


\begin{tabular}{|l|l|}
\hline Ullat & poor \\
\hline & same \\
\hline
\end{tabular}

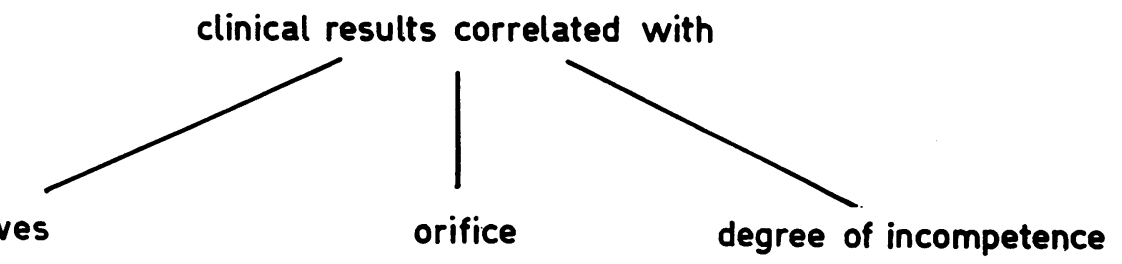

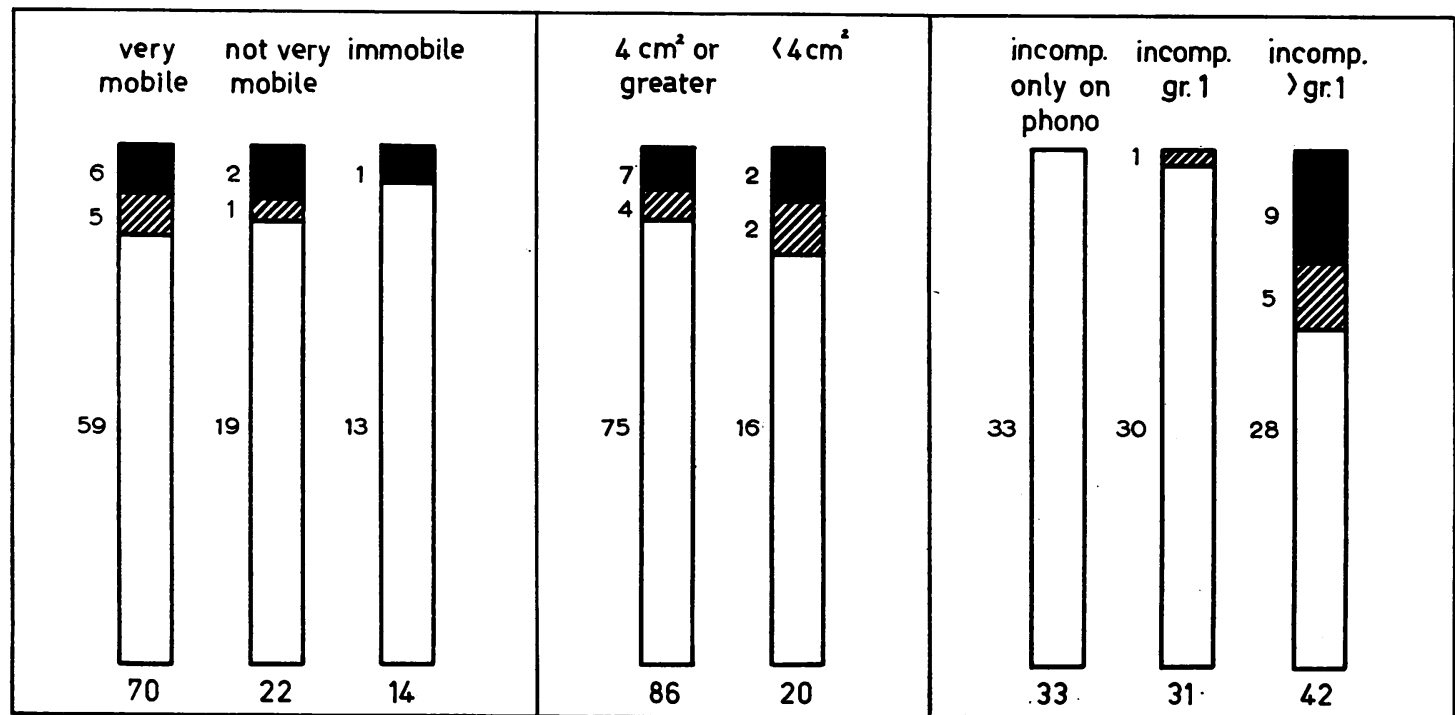

no correlation

no correlation

correlation

FIG. 7. - Diagram showing resalts of follow-up on all patients showing post-commissurotomy incompetence (sometimes only phonocardiographically demonstrable, but usually observed while the operation was still in progress.)

The group investigated included all patients who underwent an operation one to seven years previously and were known to have some degree of incompetence. The only patients excluded were those in whom an important tricuspid or aortic lesion existed in addition, because this additional anomaly may have partly determined the further clinical course. The group consisted of 106 patients. Subsequent examination at the outpatient clinic showed that the post-operative clinical condition was better than the pre-operative condition in 91 (estimated as suggested by the New York Heart Association); nine had remained unchanged and six showed deterioration. The columns in the left-hand section of Fig. 7 show that no unmistakable correlation exists between the late results and valvular mobility. If anything were to be deduced, it would be that unfavourable results are more frequently seen in patients with very mobile than in those with completely immobile valves! Nor did it seem to be essential to the patient that the mitral orifice be $4 \mathrm{sq} . \mathrm{cm}$. or more, although this is the surgeon's aim in cases of simple commissurotomy (middle section of Fig. 7). The most important data are to be found in the right-hand section of Fig. 7. The 33 patients in the left column had only slight incompetence, which had not been palpated by the surgeon but only manifested itself in the phonocardiogram by the occurrence of an apical systolic souffle which had not existed before the operation. All these patients were clinically improved. The 31 with post-operative grade $0-1$ or grade 1 incompetence were likewise clinically improved, with the exception of one, whose post-operative clinical condition remained the same. Of the 42 patients with incompetence exceeding grade 1 , there were only 28 with improvement (right column, right-hand section). Thus, there was an unmistakable association between mitral incompetence and unfavourable clinical results: virtually all patients who showed no post-operative improvement, or exacerbation, were found to have incompetence exceeding grade 1.*

\footnotetext{
*The chance that the relation found between $>$ grade 1 incompet ence and an unfavourable clinical course is based on coincidence amounts to $1: 5,000$.
} 


\section{Discussion}

On the basis of the above findings, we believe that the use of instruments in commissurotomy should be encouraged. In the hands of an experienced surgeon, instruments make it possible to attain a wider orifice without increasing the risk of development of incompetence. It should no longer happen that a surgeon must desist from widening the mitral orifice further than $2-3 \mathrm{sq} . \mathrm{cm}$. for lack of instruments.

Our present technique is as follows. An antero-lateral or postero-lateral incision is made in the fifth intercostal space on the left side, and the valves are palpated via the left auricle. The commissures are severed digitally whenever this can be very easily done. In other cases the Tubbs dilator is used, after which the antero-lateral commissure can be severed with the aid of a Brock knife if necessary. Whenever mild incompetence occurs during the operation, the surgeon exercises caution in further severing commissures, because he knows that incompetence exceeding grade 1 entails a $1: 3$ risk that the operation fails to cause improvement or even causes exacerbation.

As yet it is not clear how the technique of commissurotomy could be further perfected. In this respect we have considered the use of extracorporeal circulation, which enables the surgeon to operate under direct vision. This would make it possible to remove calcifications and to correct incompetence. In some clinics this technique is already being used in cases of secondary stenosis and combined mitral defect and in the rare cases in which marked incompetence develops during the blind operation (Kay and Nogueira, 1960; De Jesus, Breneman, Keyes, and Lam, 1960 ; Edwards and Lyons, 1960). However, as long as the results of operations for mitral incompetence remain unsatisfactory, and as long as the use of extracorporeal circulation involves considerable difficulties of organization, the open technique of commissurotomy seems to be acceptable only in exceptional cases. We have so far had no occasion to resort to it. On the other hand, it is an established fact that any closed technique yields an unsatisfactory result in 5 to $10 \%$ of cases, which at the moment could only be improved by resorting to extracorporeal circulation.

\section{SUMMARY}

The use of instruments in commissurotomy for mitral stenosis makes it possible to create a wider orifice than can be obtained by the exclusive use of the finger.

The post-operative prognosis is not primarily dependent on the fact that even the very last part of the commissures has been severed. A factor of fundamental importance in this respect is the degree of existing incompetence. If it exceeds grade 1 , then the patient runs a $1: 3$ risk of failing to improve after the operation.

In our clinic, increasing use is being made of the Tubbs dilator. The use of the Struycken scissors should be discouraged.

Further improvement of results can only be effected by a direct-vision technique with the aid of extracorporeal circulation. Chiefly because there is as yet no entirely satisfactory operative technique for mitral insufficiency, however, this method is not very attractive for the time being.

\section{REFERENCES}

Bailey, C. P., and others (1959). Dis. Chest, 35, 435. [Report of the Section on Cardiovascular Surgery of the American College of Chest Physicians (chairman: C. P. Bailey).]

De Jesus, J. R., Breneman, G. M., Keyes, J. W., and Lam, C. R (1960). Circulation, 22, 739.

Dubost, M. C. (1954). Presse méd., 62, 253.

Edwards, W. S., and Lyons, C. (1960). Circulation, 22, 744.

Ellis, L. B., Harken, D. E., and Black, H. (1959). Ibid., 19, 803.

Gerbode, F. (1960). Ibid., 21, 563.

Kay, E. B., and Nogueira, C. (1960). J. Amer. med. Ass., 173, 1644. Logan, A., and Turner, R. (1959). Lancet, 2, 874.

New York Heart Association (1953). Nomenclature and Criteria for Diagnosis of Diseases of the Heart and Blood Vessels, 5th ed. New York.

Romanos, A. N., and Derom, F. E. (1955). Lancet, 2, 1175. 